\title{
Markedly elevated plasma D-dimer and the prevalence of acute pulmonary embolus
}

\author{
Andrew W. Bowman ${ }^{1}$, Gretchen S. Johns ${ }^{2}$, and Nolan Karstaedt ${ }^{1}$
}

${ }^{1}$ Department of Radiology, Mayo Clinic Florida, USA

${ }^{2}$ Division of Laboratory Medicine and Pathology, Mayo Clinic Florida, USA

Corresponding author: Andrew W. Bowman Department of Radiology Mayo Clinic Florida 4500 San Pablo Road Jacksonville, FL 32224 bowman.andrew@mayo.edu Tel.: + 904 953-2000

Fax: + 904 953-1044

Received: 18 July 2011

Accepted: 8 November 2011

Copyright (C) 2011 by

Academy of Sciences and Arts of Bosnia and Herzegovina. E-mail for permission to publish: amabih@anubih.ba
Objective. To evaluate if increasingly elevated levels of plasma Ddimer are associated with higher prevalence of acute pulmonary embolus (PE). Patients and Methods. A retrospective study was conducted evaluating all PE protocol CT examinations performed in low-to-intermediate risk emergency department and hospitalized patients during 2007. All PE protocol CT reports were reviewed for the presence or absence of acute PE. The charts of all of these subjects were then reviewed for quantitative plasma $\mathrm{D}$-dimer values, measured in $\mathrm{mg} / \mathrm{ml}$ Fibrinogen Equivalent Units, drawn within one day prior to the CT exam. The prevalence of acute PE at different D-dimer threshold results was then evaluated using D-dimer groups as follows: $<1.0$ $\mathrm{mg} / \mathrm{ml}, \geq 1.0$ but $<2.0 \mathrm{mg} / \mathrm{ml}, \geq 2.0$ but $<4.0 \mathrm{mg} / \mathrm{ml}$, and $\geq 4.0 \mathrm{mg} /$ ml. Results. 943 PE protocol CT exams were reviewed. 410 subjects had D-dimer values drawn before their CT exams; 30 (7.3\%) of these were positive for acute $\mathrm{PE}$. As D-dimer values became increasingly elevated, the prevalence of acute PE increased accordingly. In particular, D-dimer elevation $\geq 4.0 \mathrm{mg} / \mathrm{ml}$ was almost $94 \%$ specific for acute PE by CT criteria. Conclusion. Increased elevation of plasma D-dimer is associated with increased prevalence of acute PE in low-to-intermediate risk patients.

Key words: Pulmonary embolus, D-dimer, CT.

\section{Introduction}

Acute pulmonary embolism (PE) remains a critical diagnosis, and the need for rapid diagnosis of acute PE is similarly critical. The current standard diagnostic method for diagnosing acute $\mathrm{PE}$ is through the use of multidetector contrast-enhanced CT using thin-slice reconstructions (PE protocol CT) (1). Various clinical algorithms for the evaluation for acute PE exist $(2,3)$. These algorithms, in conjunction with plasma $\mathrm{D}$-dimer values, are frequently used to help classify patients as high or low risk for acute PE. Prior studies demonstrate that "normal" values of D- 
dimer within the reference interval predict successfully a very low probability of thromboembolic disease and effectively exclude acute $\mathrm{PE}$ in low-to-intermediate risk patients. However, as it is known to be elevated in a variety of conditions, borderline elevated $\mathrm{D}$-dimer levels are not very specific for the presence of PE (4). To our knowledge, there have been no recent studies evaluating the specificity of markedly elevated plasma $\mathrm{D}$-dimer levels for the presence of acute PE.

The aim of this study is to evaluate the prevalence of acute PE by CT criteria in lowto-intermediate risk hospitalized and emergency department (ED) patients with markedly increased plasma D-dimer values; specifically, if acute PE prevalence is higher in such patients with greater $\mathrm{D}$-dimer values.

\section{Patients and methods}

\section{Patients and data collection}

After approval from our Institutional Review Board, and in compliance with the Health Insurance Portability and Accountability Act, a retrospective review of all reports from CT examinations performed at a single urban teaching hospital between January 1, 2007 and December 31, 2007 was conducted to search for PE protocol CT exams. The PE protocol CT exams were found by searching the radiology CT report database using the keywords "PE", "pulmonary embolus", and "pulmonary emboli". Of the retrieved reports, only those for PE protocol CT exams were considered; reports from aortic dissection protocol CT or standard contrast enhanced chest CT examinations that incidentally discovered PEs were excluded. Studies performed in patients under the age of 18 and those performed on a strict outpatient basis were also excluded. The collected reports were then reviewed for the presence or absence of acute PE. A report was considered positive for acute $\mathrm{PE}$ if (1) at least one pulmonary embolus was present, and (2) there was no description in the report to suggest that the embolus had been present on a prior examination or was chronic in appearance. Any limitations to the diagnostic quality of the CT exams noted in the radiology reports (i.e., patient respiratory motion, poor opacification of the pulmonary arteries, etc) were also tabulated.

\section{Imaging}

For the data included in this study, PE protocol CT exams were performed on 16 channel and 4 channel multidetector CT scanners (Siemens Medical Solutions, Erlangen, Germany). All images were reconstructed to $2 \mathrm{~mm}$ slices for interpretation on a PACS workstation.

A volume of 75-150 ml low-osmolar iodinated contrast material (iohexol, Omnipaque 300, GE Healthcare, Waukesha, WI, USA) or iso-osmolar iodinated contrast material (iodixanol, Visipaque, GE Healthcare) was administered at $4 \mathrm{ml} / \mathrm{s}$ via a power injector (MEDRAD, Inc, Warendale, PA, USA). A region of interest was placed over the main pulmonary artery, and image acquisition was triggered once the average density within the region of interest exceeded a threshold of 90 Hounsfield Units (bolus tracking). All exams were interpreted by board certified radiologists, and the documented PE protocol CT result as described above was based on this reading.

\section{D-dimer data collection}

Once all the PE protocol CT reports were obtained, the charts of all subjects were reviewed for all available $\mathrm{D}$-dimer testing. Any D-dimer values obtained on the day of or the day prior to the PE protocol CT examination were documented. Our institution utilizes a commercially available, automated, immuno-turbidimetric assay with latex microparticles (Diagnostica Stago, Paris, France) which provides fully quantitative 
$\mathrm{D}$-dimer values between the ranges of 0.22 and $20.0 \mu \mathrm{g} / \mathrm{ml}$. The upper limit of normal for D-dimer values (in Fibrinogen Equivalent Units or FEU) at our institution in 2007 was $0.4 \mu \mathrm{g} / \mathrm{ml}$. Internal validation of this $\mathrm{D}$ dimer assay performed in 2003 at our institution in normal adults $(\mathrm{n}=62)$ yielded (in $\mu \mathrm{g} / \mathrm{ml})$ : mean $=0.30 ; 1$ standard deviation $=$ $0.10 ; 2$ standard deviations $=0.21 ;$ median $=$ 0.29 ; range $=0.15-0.50$.

\section{Statistical analysis}

Once all data were tabulated, the sensitivity, specificity, positive predictive value, negative predictive value, and accuracy of the ability of $\mathrm{D}$-dimer to predict the presence of acute $\mathrm{PE}$ on $\mathrm{CT}$ were calculated at $\mathrm{D}$-dimer values of $<1.0 \mu \mathrm{g} / \mathrm{ml}, \geq 1.0$ but $<2.0 \mu \mathrm{g} / \mathrm{ml}, \geq 2.0$ but $<4.0 \mu \mathrm{g} / \mathrm{ml}$, and $\geq 4.0 \mu \mathrm{g} / \mathrm{ml}$. Appropriate $95 \%$ confidence intervals were also generated. Pearson's $\chi^{2}$ analysis was employed to test the null hypothesis that prevalence of acute $\mathrm{PE}$ was independent of $\mathrm{D}$-dimer value. A p-value of $<0.05$ was considered statistically significant.

\section{Results}

\section{Acute PE prevalence}

962 PE protocol examinations were performed in our hospital in 2007. One examination was excluded from this study as the patient was under 18 years of age, and 18 additional examinations performed on nonemergency outpatients were also excluded. Therefore, 943 CT examinations, from a total number of 883 subjects ( 343 male; ages ranging from 18 to 99 years), were included in the final analysis. All subjects were either inpatients or ED patients. 87 examinations (9\%) were positive for acute pulmonary embolus. Of the $943 \mathrm{CT}$ exams included, 410 from a total of 403 subjects (146 male, ages ranging from 18 to 99 years) also had accompanying $\mathrm{D}$-dimer values drawn within the specified time interval $(43 \%$ of all $\mathrm{PE}$ protocol CTs). Of these exams, 30 (7.3\%) were positive for acute PE (Table 1).

Table 1 Prevalence of acute PE in subjects with and without $\mathrm{D}$-dimer values obtained.

\begin{tabular}{lccc}
\hline \multirow{2}{*}{ D-dimer Data } & \multicolumn{2}{c}{ D-dimer } & Total \\
\cline { 2 - 3 } & Drawn & Not drawn & \\
\hline $\begin{array}{l}\text { PE CT Exams } \\
\text { Performed (n) }\end{array}$ & 410 & 533 & 943 \\
$\begin{array}{l}\text { Acute pulmonary } \\
\text { embolus (n) }\end{array}$ & 30 & 57 & 87 \\
$\begin{array}{l}\text { Acute pulmonary } \\
\text { embolus } \\
\text { prevalence (\%) }\end{array}$ & 7.3 & 10.7 & 9.2 \\
$\begin{array}{l}\text { 95\% confidence } \\
\text { intervals }\end{array}$ & $4.8-9.8$ & $8.1-13.3$ & $7.4-11.1$ \\
\hline
\end{tabular}

56 subjects underwent more than one $\mathrm{PE}$ protocol CT examination during 2007 (a total of 116 exams). Accompanying D-dimer values were drawn for 40 of these 116 exams. 18 of the 116 exams were positive for acute $\mathrm{PE}$, and 5 subjects had acute PE on multiple exams. Only 1 follow-up PE protocol CT in which the initial exam demonstrated acute PE had an accompanying $\mathrm{D}$-dimer value. In this particular case, the follow-up CT was negative for acute $\mathrm{PE}$, and the accompanying D-dimer value was $0.22 \mu \mathrm{g} / \mathrm{ml}$.

Of the 943 total exams, 117 of the reports described some limitation to the imaging. The type of limitation most cited was subject respiratory motion (96 exams). However, only 1 exam was deemed non-diagnostic (an exam that did not have an accompanying Ddimer value).

\section{Prevalence stratified by $D$-dimer value}

Using the findings on CT as the reference standard, the sensitivities, specificities, and positive and negative predictive values for using the stated D-dimer values are listed in Table 2.

As $\mathrm{D}$-dimer values increase, the prevalence of acute $\mathrm{PE}$ also increases in a statistically significant manner $\left(\mathrm{p}<0.05, \chi^{2}=55.07\right.$, 
Table 2 Acute pulmonary embolus at different D-dimer thresholds

\begin{tabular}{llll}
\hline \multirow{2}{*}{ Reliability } & \multicolumn{2}{l}{ D-dimer Threshold Value $(\mathrm{mg} / \mathrm{ml})$} \\
\cline { 2 - 4 } & 1.0 & 2.0 & 4.0 \\
\hline Sensitivity (\%; 95\%Cl) & 96.7 & 80.0 & 46.7 \\
& $(95.0-98.4)$ & $(76.1-83.9)$ & $(41.9-51.5)$ \\
Specificity (\%; 95\%Cl) & 56.6 & 78.4 & 93.9 \\
& $(51.8-61.4)$ & $(74.4-82.4)$ & $(91.6-96.2)$ \\
Positive predictive value (\%; 95\%Cl) & 14.9 & 22.6 & 37.8 \\
& $(11.5-18.3)$ & $(18.6-26.6)$ & $(33.1-42.5)$ \\
Negative predictive value (\%; 95\%Cl) & 99.5 & 98.0 & 95.7 \\
& $(98.8-100.0)$ & $(96.6-99.4)$ & $(93.7-97.7)$ \\
Accuracy (\%; 95\%Cl) & 59.5 & 78.5 & 90.5 \\
& $(54.7-64.3)$ & $(74.5-82.5)$ & $(87.7-93.3)$ \\
\hline
\end{tabular}

$\mathrm{Cl}=$ Confidence interval .

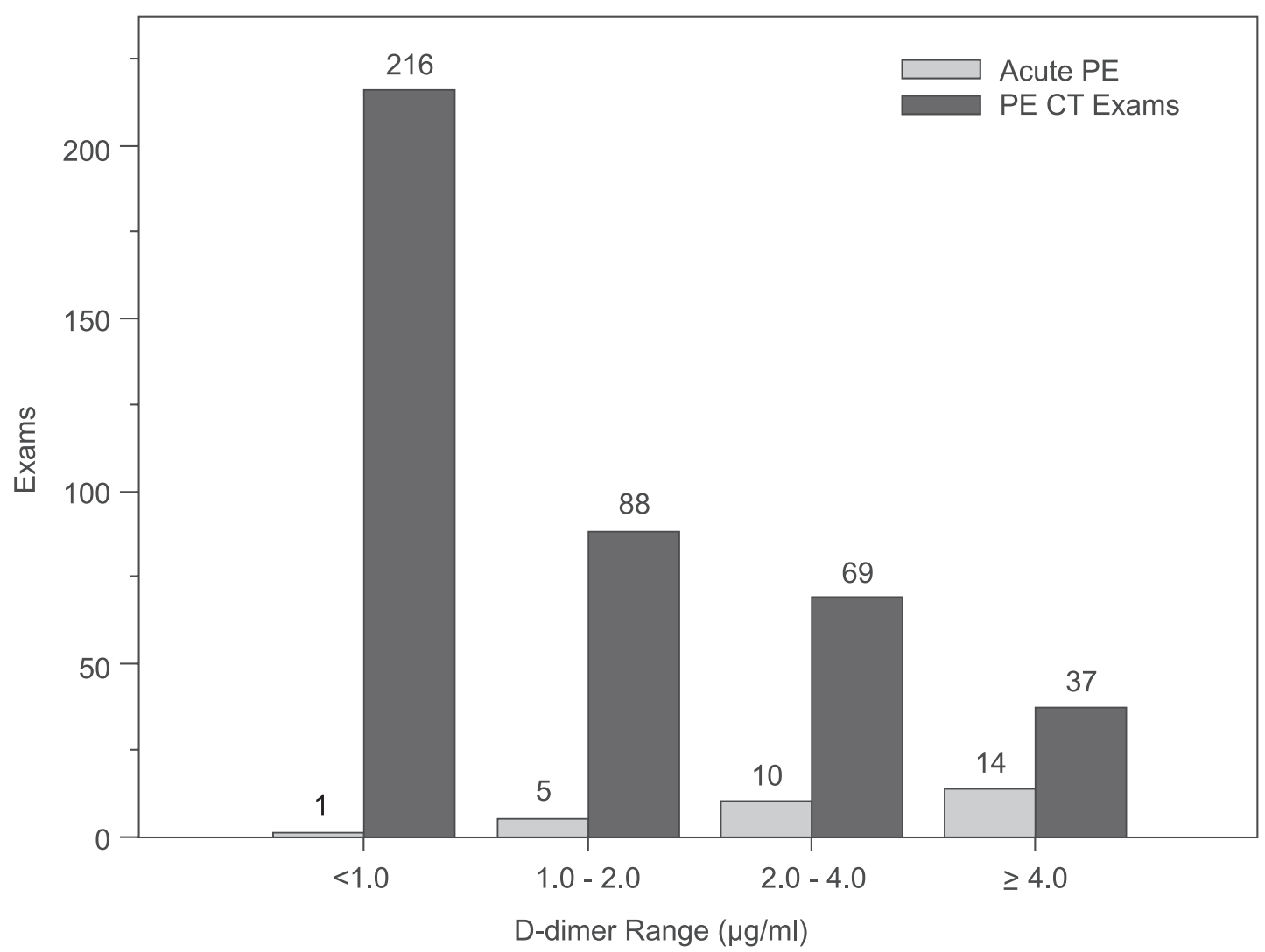

Figure 1 Distribution of acute PE studies according to D-dimer values for the 410 subjects in whom both D-dimer values and a PE protocol CT were obtained. Above each bar is the actual value from which the bar is generated (i.e., when D-dimer $<1.0 \mu \mathrm{g} / \mathrm{ml}$, there was 1 study positive for acute PE out of a total of 216 studies). Note that studies in which the D-dimer value equaled exactly $1.0 \mu \mathrm{g} / \mathrm{ml}$ are grouped in the "1.0-2.0" bin and those in which the D-dimer value equaled exactly $2.0 \mu \mathrm{g} / \mathrm{ml}$ are grouped in the "2.0-4.0" bin. See text for details. 
3 degrees of freedom). Importantly, more than one-third of subjects with D-dimer values exceeding $4.0 \mu \mathrm{g} / \mathrm{ml}$ had acute $\mathrm{PE}$ (Figure 1).

\section{Discussion}

Our study retrospectively evaluated the relationship between the degree of $\mathrm{D}$-dimer elevation and the prevalence of acute PE in low-to-intermediate risk hospitalized and ED patients. The overall prevalence of acute $\mathrm{PE}$ in this population is slightly higher than in those of other recent studies (5-7). A reason for this may be that, in contrast to these prior studies that evaluated only ED patients, our study also included PE protocol CT exams performed on inpatients, who may be at increased risk for PE relative to the ED population.

A well-documented shortcoming of the $\mathrm{D}$-dimer assay in the investigation of acute $\mathrm{PE}$ has been its low specificity, as D-dimer values may be elevated in a number of other physiological and pathophysiological states (4). Our results suggest that as D-dimer values increase, they become much more specific for the presence of acute PE, reaching almost $94 \%$ specificity when D-dimer values exceed $4.0 \mu \mathrm{g} / \mathrm{ml}$. This may be potentially helpful in patients who are unable to undergo a PE protocol CT because of decreased renal function or contrast allergy, though this requires validation in additional studies. Further evaluation in more subjects with markedly elevated D-dimer levels and their correlation with other measures of PE (i.e. nuclear medicine ventilation/perfusion scans and/or lower extremity Doppler ultrasound exams) would also be helpful in optimizing the management of such patients.

We note that the D-dimer threshold levels used in this study are significantly higher than the upper limit of normal, both employed by our institution $(0.4 \mu \mathrm{g} / \mathrm{ml})$ and recommended by the most comprehensive recent review $(0.5 \mu \mathrm{g} / \mathrm{ml})(4)$. The purpose of this study is not to evaluate the appropriate threshold for excluding acute PE, but to determine whether there is a relationship between the degree of $\mathrm{D}$-dimer elevation and the prevalence of acute PE. However, it has not escaped our attention that acute $\mathrm{PE}$ prevalence in low-to-intermediate risk patients with lower $\mathrm{D}$-dimer values is exceedingly rare ( 1 out of 216 in patients with $\mathrm{D}$-dimer values $<1.0 \mu \mathrm{g} / \mathrm{ml}$ ). In fact, none of the subjects with $\mathrm{D}$-dimer values $<0.4 \mu \mathrm{g} /$ $\mathrm{ml}$ ( 43 exams) or $<0.5 \mu \mathrm{g} / \mathrm{ml}$ ( 80 exams) had acute PE; the one positive case for acute $\mathrm{PE}$ with $\mathrm{D}$-dimer $<1.0 \mu \mathrm{g} / \mathrm{ml}$ had a value of 0.62 $\mu \mathrm{g} / \mathrm{ml}$. Additional recent studies suggest that $\mathrm{D}$-dimer threshold values higher than $0.5 \mu \mathrm{g} / \mathrm{ml}$ may safely exclude acute PE (7-9), which our data support.

\section{Limitations}

This is a retrospective study and is therefore subject to biases inherent in all such studies. $\mathrm{D}$-dimer results would frequently have been available to the radiologist interpreting the $\mathrm{CT}$ at the time of its interpretation, so some element of bias in the interpretation is impossible to exclude. Patients at low-to-intermediate risk for acute PE that were unable to receive a PE protocol CT were not included in this study, and this may contribute to an additional selection bias. No correlation was made with the presence or absence of deep venous thrombosis as a possible explanation for an elevated D-dimer, though we reason that if the patients did not present with pulmonary and/or chest pain symptoms, they would not have been evaluated for acute PE using CT in the first place. There were 57 patients with acute PE who did not have Ddimer values drawn. However, if the clinical suspicion for acute PE is sufficiently great, checking a D-dimer value first would have been generally inappropriate if not unsafe $(3,5,6,10)$. However, having more cases of acute $\mathrm{PE}$ with correlating $\mathrm{D}$-dimer values 
would be helpful, particularly cases with markedly elevated $\mathrm{D}$-dimer values. The decision to group ED patients and inpatients together in our study population was made largely due to the fact that many inpatients had some component of chest pain at their initial presentation to the ED. PE protocol CT exams were often ordered by admitting physicians while the patient was still in the $\mathrm{ED}$ or by the ED physician after the decision to admit had already been made, making appropriate segregation of these patients difficult. Additionally, different institutions use different D-dimer tests with different units (ELISA vs. latex turbidometric, plasma vs. whole blood, FEUs vs. D-dimer units, $\mu \mathrm{g} /$ $\mathrm{ml}, \mu \mathrm{g} / \mathrm{l}, \mathrm{ng} / \mathrm{ml}$ or $\mathrm{mg} / \mathrm{l}$ ), and caution must be used when comparing results and numerical values of $\mathrm{D}$-dimer tests. And finally, while CT findings were used as the standard for the presence or absence of acute PE in this study, it is important to remember that despite being the current method of choice for diagnosis, PE protocol CT is not $100 \%$ sensitive for identification of all PEs; indeed, many prior studies report a sensitivity of $<90 \%$, particularly for smaller, more distal emboli (11).

\section{Conclusion}

The prevalence of acute PE by CT criteria in low-to-intermediate risk patients increases as plasma D-dimer values become increasingly elevated. In particular, markedly increased D-dimer values are highly specific for acute $\mathrm{PE}$ in this population, and such degrees of elevation may be helpful in the management of patients at risk for acute $\mathrm{PE}$ who cannot undergo PE protocol CT.

\section{Acknowledgements}

We are grateful to Dallas Thorpe for technical expertise on PE protocol CT technique, Rebecca McNeil and Michael Heckman for discussion on statistics, and Barbara McComb and Margaret Johnson for assistance with manuscript preparation.
Conflict of interest: The authors declare that they have no conflict of interest. This study was not sponsored by any external organization.

Authors' contributions: Conception and design: $\mathrm{AWB}$ and NK; Acquisition, analysis and interpretation of data: AWB; Drafting the article AWB and GSJ, Revising it critically for important intellectual content: AWB, GSJ, and NK.

\section{References}

1. Remy-Jardin M, Remy J, Deschildre F, Artaud D, Beregi JP, Hossein-Foucher C, et al. Diagnosis of pulmonary embolism with spiral CT: comparison with pulmonary angiography and scintigraphy. Radiology. 1996;200:699-706.

2. Wells PS, Ginsberg JS, Anderson DR, Kearon C, Gent M, Turpie AG, et al. Use of a clinical model for safe management of patients with suspected pulmonary embolism. Ann Intern Med. 1998;129:997-1005.

3. Wells PS, Anderson DR, Rodger M, Ginsberg JS, Kearon C, Gent M, et al. Derivation of a simple clinical model to categorize patients probability of pulmonary embolism: increasing the models utility with the SimpliRED D-dimer. Thromb Haemost. 2000;83:416-20.

4. Stein PD, Hull RD, Patel KC, Olson RE, Ghali WA, Brant R, et al. D-dimer for the exclusion of acute venous thrombosis and pulmonary embolism: a systematic review. Ann Intern Med. 2004;140:589602.

5. Gupta RT, Kakarla RK, Kirshenbaum KJ, Tapson VF. D-dimers and efficacy of clinical risk estimation algorithms: sensitivity in evaluation of acute pulmonary embolism. AJR Am J Roentgenol. 2009;193:425-30.

6. Corwin MT, Donohoo JH, Partridge R, Egglin TK, Mayo-Smith WW. Do emergency physicians use serum D-dimer effectively to determine the need for CT when evaluating patients for pulmonary embolism? Review of 5,344 consecutive patients. AJR Am J Roentgenol. 2009;192:1319-23.

7. Hirai LK, Takahashi JM, Yoon HC. A prospective evaluation of a quantitative D-dimer assay in the evaluation of acute pulmonary embolism. J Vasc Interv Radiol. 2007;18:970-4.

8. Gimber LH, Travis RI, Takahashi JM, Goodman TL, Yoon HC. Computed Tomography Angiography in Patients Evaluated for Acute Pulmonary Embolism with Low Serum D-dimer Levels: A Prospective Study. Perm J. 2009;13:4-10. 
9. Sanchez LD, McGillicuddy DC, Volz KA, Fan SL, Joyce N, Horowitz GL. Effect of two different FDA-approved D-dimer assays on resource utilization in the emergency department. Acad Emerg Med. 2011;18(3):317-21.

10. Weiss CR, Haponik EF, Diette GB, Merriman B, Scatarige JC, Fishman EK. Pretest risk assessment in suspected acute pulmonary embolism. Acad Radiol. 2008;15:3-14.

11. Eng J, Krishnan JA, Segal JB, Bolger DT, Tamariz LJ, Streiff MB, et al. Accuracy of CT in the diagnosis of pulmonary embolism: a systemic literature review. AJR Am J Roentgenol. 2004;183:1819-27. 